\title{
Filming movies of attosecond charge migration with high harmonic spectroscopy
}

\section{Pengfei Lan ( $\nabla$ pengfeilan@hust.edu.cn)}

Wuhan National Laboratory for Optoelectronics and School of Physics, Huazhong University of Science and Technology

\section{Lixin He}

\section{Siqi Sun}

Wuhan National Laboratory for Optoelectronics and School of Physics, Huazhong University of Science and Technology

\section{Yanqing He}

Wuhan National Laboratory for Optoelectronics and School of Physics, Huazhong University of Science and Technology

\section{Bincheng Wang}

Wuhan National Laboratory for Optoelectronics and School of Physics, Huazhong University of Science and Technology

\section{Pu Wang}

Wuhan National Laboratory for Optoelectronics and School of Physics, Huazhong University of Science and Technology

\section{Xiaosong Zhu}

Wuhan National Laboratory for Optoelectronics and School of Physics, Huazhong University of Science and Technology

\section{Liang Li}

Wuhan National Laboratory for Optoelectronics and School of Physics, Huazhong University of Science and Technology

\section{Wei Cao}

Wuhan National Laboratory for Optoelectronics and School of Physics, Huazhong University of Science and Technology

\section{Peixiang Lu}

Huazhong University of Science and Technology https://orcid.org/0000-0001-6993-8986

\section{Chii-Dong Lin}

Kansas State University https://orcid.org/0000-0003-4847-8938

\section{Physical Sciences - Article}

Keywords: high-harmonic spectroscopy, ultrafast charge migration 
Posted Date: March 2nd, 2021

DOI: https://doi.org/10.21203/rs.3.rs-185507/v1

License: (c) (i) This work is licensed under a Creative Commons Attribution 4.0 International License. Read Full License 


\title{
Filming movies of attosecond charge migration with high harmonic spectroscopy
}

\author{
Lixin $\mathrm{He}^{1, *}$, Siqi Sun ${ }^{1, *}$, Pengfei $\mathrm{Lan}^{1, \dagger}$, Yanqing $\mathrm{He}^{1}$, Bincheng Wang ${ }^{1}, \mathrm{Pu}$ \\ Wang ${ }^{1}$, Xiaosong Zhu ${ }^{1}$, Liang $\mathrm{Li}^{1}$, Wei Cao ${ }^{1}$, Peixiang $\mathrm{Lu}^{1,2, \ddagger}$, and C. D. $\operatorname{Lin}^{3}$ \\ ${ }^{1}$ Wuhan National Laboratory for Optoelectronics and School of Physics, \\ Huazhong University of Science and Technology, \\ Wuhan 430074, China \\ ${ }^{2}$ CAS Center for Excellence in Ultra-intense Laser Science, \\ Shanghai 201800, China \\ ${ }^{3}$ Department of Physics, Cardwell Hall, \\ Kansas State University, \\ Manhattan, KS 66506, USA
}

(Dated: January 29, 2021)

Ultrafast electron migration in molecules is the progenitor of all chemical reactions and biological functions after light-matter interaction [1-4]. Following this ultrafast dynamics, however, has been an enduring endeavor $[5,6]$. Recently, it has been shown that high-harmonic spectroscopy (HHS) is able to probe dynamics with attosecond temporal and sub-ångström spatial resolution [7-10]. Still, real-time visualization of single-molecule dynamics continues to be a great challenge because experimental harmonic spectra are due to the coherent averages of light emission from individual molecules of different alignments. Here, we show that from high harmonics generated with single-color and two-color probe lasers in a pump-probe experiment, the complex amplitude and phase of harmonics from a single fixed-in-space molecule can be reconstructed using modern machine learning (ML) algorithm. From the complex single-molecule dipoles for different harmonics, we construct a series of film clips of hole density distributions of the cation at time steps of 50 attoseconds $\left(1 \mathrm{as}=10^{-18} \mathrm{~s}\right)$ to make a classical "movie" of electron migration after tunnel ionization of the molecule. Moreover, the angular dependence of molecular charge migration is fully resolved. By examining these clips, we observed that holes do not just "migrate" along the laser direction, but they may "swirl" around the atom centers. The

\footnotetext{
* These authors contributed equally to this work.

† pengfeilan@hust.edu.cn

‡ lupeixiang@hust.edu.cn
} 


\begin{abstract}
ML-based HHS proposed here establishes a general reconstruction scheme for studying ultrafast charge migration in molecules, paving a way for further advance in tracing and controlling photochemical reactions by femtosecond lasers.
\end{abstract}

\title{
I. MAIN
}

High harmonic spectroscopy (HHS) relies on an "internal" built-in pump-probe process in highorder harmonic generation (HHG) known as a three-step model $[11,12]$ : ionization (pump), acceleration, and recombination (probe) [see Fig. 1(a)]. In this process, the electron dynamics is triggered at the ionization step, and probed at the recombination instant by the returning electron with information encoded in the harmonic spectrum. The temporal resolution of this unique pump-probe process arises from the intrinsic frequency chirp of harmonic emission [13], i.e., different harmonic orders are associated with well-defined ionization-recombination delays spent by the electron in the continuum [see Fig. 1(b)]. The typical resolution for a commonly-used $800 \mathrm{~nm}$ driving laser is sub-100 as. The spatial resolution of HHS comes from the de Broglie wavelength of the recombining electron and can reach sub-ångström [14]. With this technique, measurement of ultrafast nuclear wave packets created by ionization in isotopic molecules was realized $[15,16]$. Very recently, the HHS technique has been further nicely extended to extract attosecond charge migration in iodoacetylene molecule [17].

As demonstrated in [17], to reconstruct electron dynamics of single molecules from experimental harmonic spectra, one has to address many challenges. First, reconstruction is an inverse scattering problem, which is generally solved by iterative methods. Reconstruction is possible only if the scattering theory is on firm ground. Second, since molecules can not be aligned perfectly in experiment, the measured harmonics are the result of a coherent superposition of individual radiation weighted by the angular distribution of the molecules [18-20]. Thus, the critical challenge is to extract fixed-in-space single-molecule harmonic amplitudes and phases. Besides, to extract charge migration, extra parameters that describe electron dynamics should be obtained as well. Thus, it is also necessary to evaluate if adequate experimental data are available and accurate retrieval of charge migration is possible.

Here we demonstrate that, by resolving the above three issues, we have succeeded in filming movies of charge migration in the molecular ion using HHS to reveal substantial sub-ångström electron migration. Since electron dynamics runs on the timescale of about 20 as (one atomic unit of time is 24 as), while the emission time difference between two odd harmonics by an 800-nm 

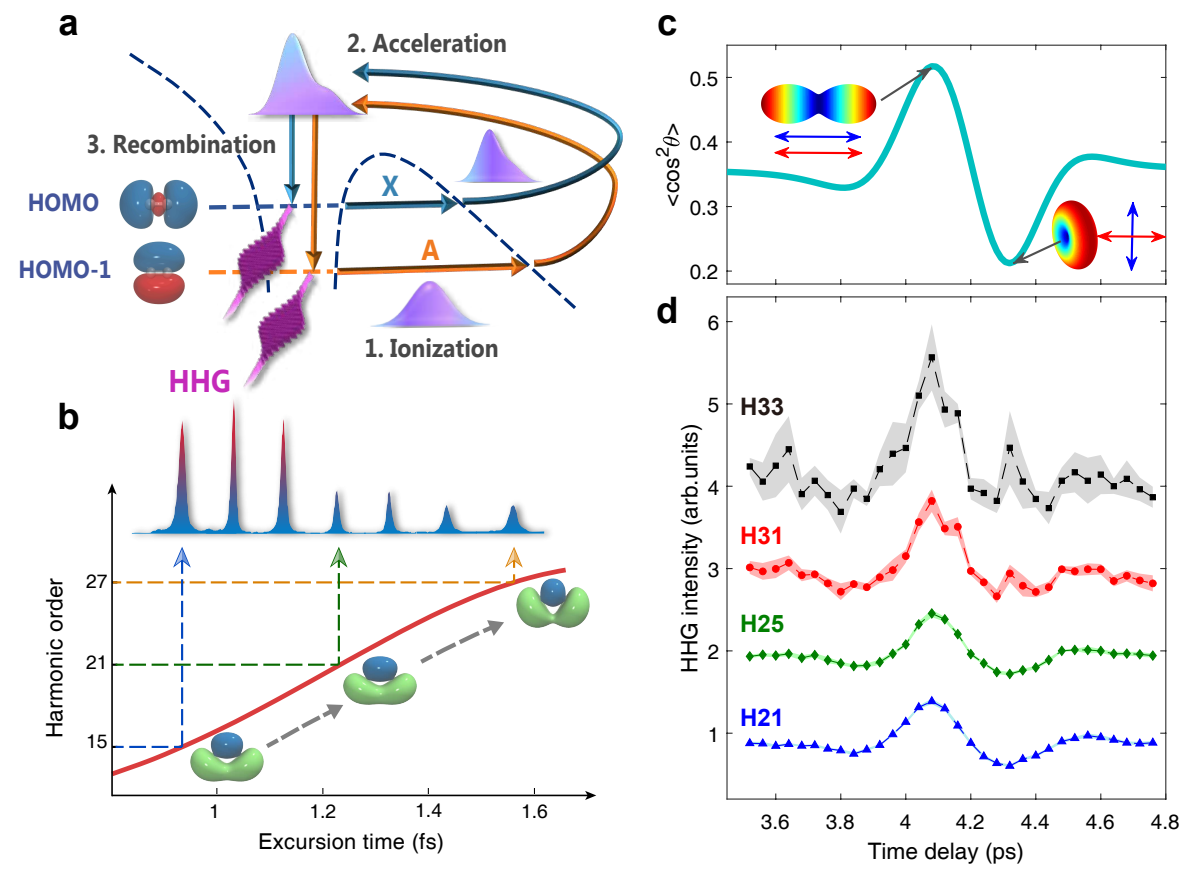

FIG. 1: Harmonic generation from $\mathbf{N}_{2}$ molecule. a, HHG from each molecule can be described by the semi-classical three-step model : (1) ionization, (2) acceleration, and (3) recombination. After ionization, the molecular system is usually left in ground and several electronically excited states of the ion. In the HHG process, these ionic states provide different channels (Channels $X$ and $A$ for $\mathrm{N}_{2}$ ) that connect the same initial and final states of the system. The interference of harmonics of these channels leads to the eventual harmonic emission. b, Time-frequency mapping in the HHG process. For different high-harmonic orders, the freed electron spends different excursion times (the time interval between the ionization and recombination of the electron) in the continuum, providing a one-to-one mapping between time and harmonic photon energy to enable temporal measurement of charge migration underlying the HHG process. Here we show only harmonics from the short-trajectory electrons due to its better phase matching in our experiment. c, Time-dependent degrees of molecular alignment $\left[\left\langle\cos ^{2} \theta\right\rangle(\mathrm{t})\right]$ near the half rotational revival of $\mathrm{N}_{2}$ in our experiment. The embedded false-color plots with prolate and oblate shapes correspond to molecular angular distributions at maximal alignment (4.08 ps) and maximal anti-alignment (4.32 ps) moments, respectively. Blue and red arrows indicate the directions of molecular alignment and the polarization of the driving laser pulse. d, Measured HHG signals of H21, H25, H31 and H33 of $\mathrm{N}_{2}$ at the pump-probe delays in c. For clarity, the harmonic intensities here have been normalized to results measured for the isotropic case (i.e., without the alignment pulse). The results of H25, H31 and H33 have been shifted vertically. Shaded areas in $\mathbf{d}$ represent the standard deviation of the signals averaged over 1000 laser shots. The different spectral features of H31 and H33 from lower harmonics are due to contribution from the HOMO-1 orbital, especially at the time of maximal anti-alignment. 
laser is about 100 as, we shorten this interval by half, by generating both even and odd harmonics with a two-color laser field. With harmonic spectra from both single-color and two-color lasers in standard pump-probe measurements, together with the powerful modern machine learning (ML) algorithm, we have abundant data to retrieve the complex dipole amplitude and phase of each fixed-in-space molecule to construct charge migration movies.

\section{Deciphering charge migration with HHS}

We first explain how ultrafast charge migration is encoded in and how it can be identified from the molecular HHG signals. After strong-field tunneling ionization, the molecule in general is left in the ground as well as several nearby electronically excited states of the ion [1-4, 21-23]. Once the electron is ejected, the occupation amplitudes of these electronic states would change with time under the influence of the external laser field, creating a time-dependent many-electron wave packet, or equivalently, an electron hole wave packet. The time dependence of the modulus square of the wave packet is called electron charge (or hole) migration. Such hole dynamics is encoded in the harmonic spectra of each single molecule [21-23].

To decipher charge migration from the harmonic spectra, we choose the two most widely studied molecules $\mathrm{N}_{2}$ and $\mathrm{CO}_{2}$ as the prototypes to demonstrate our scheme. First, we consider $\mathrm{N}_{2}$. As illustrated in Fig. 1(a), the two channels (Channel $X$ and $A$ ) relevant to HHG in $\mathrm{N}_{2}$ are associated with the ground $\tilde{X}^{2} \Sigma_{g}$ and first excited $\tilde{A}^{2} \Pi_{u}$ states of $\mathrm{N}_{2}^{+}$ion. In molecular orbital picture, these two channels correspond to ionization of an electron from the two highest-occupied molecular orbitals, HOMO and HOMO-1, respectively. Owing to the different symmetries of these orbitals, HHG from different ionization channels depends differently on molecular alignment. Figure 1(c) depicts the typical alignment distributions of $\mathrm{N}_{2}$ molecules near the half revival period, while Figure 1(d) shows the typical harmonic spectra measured in experiment (for experimental details, see Methods and Supplementary Note 1). When molecules are aligned (anti-aligned) with respect to laser polarization, the signals of lower order harmonics (e.g., H21 and H25) are strong (weak) because they are generated from the HOMO orbital. For higher orders, like H31 and H33, the signals receive substantial contribution from the HOMO-1 orbital and thus becomes quite large when the molecules are anti-aligned. These properties of the harmonic spectra of $\mathrm{N}_{2}$ have been well studied experimentally [24] and can be calculated with quantitative rescattering (QRS) theory [2528]. Similar results are also observed in HHG from $\mathrm{CO}_{2}$ molecule (see Supplementary Note 2). The different alignment dependence of the contribution to HHG from multiple molecular orbitals allows us to access their complex population amplitudes in the complex wave packet of the molecular ion, 
from which ultrafast charge migration can be uncovered.
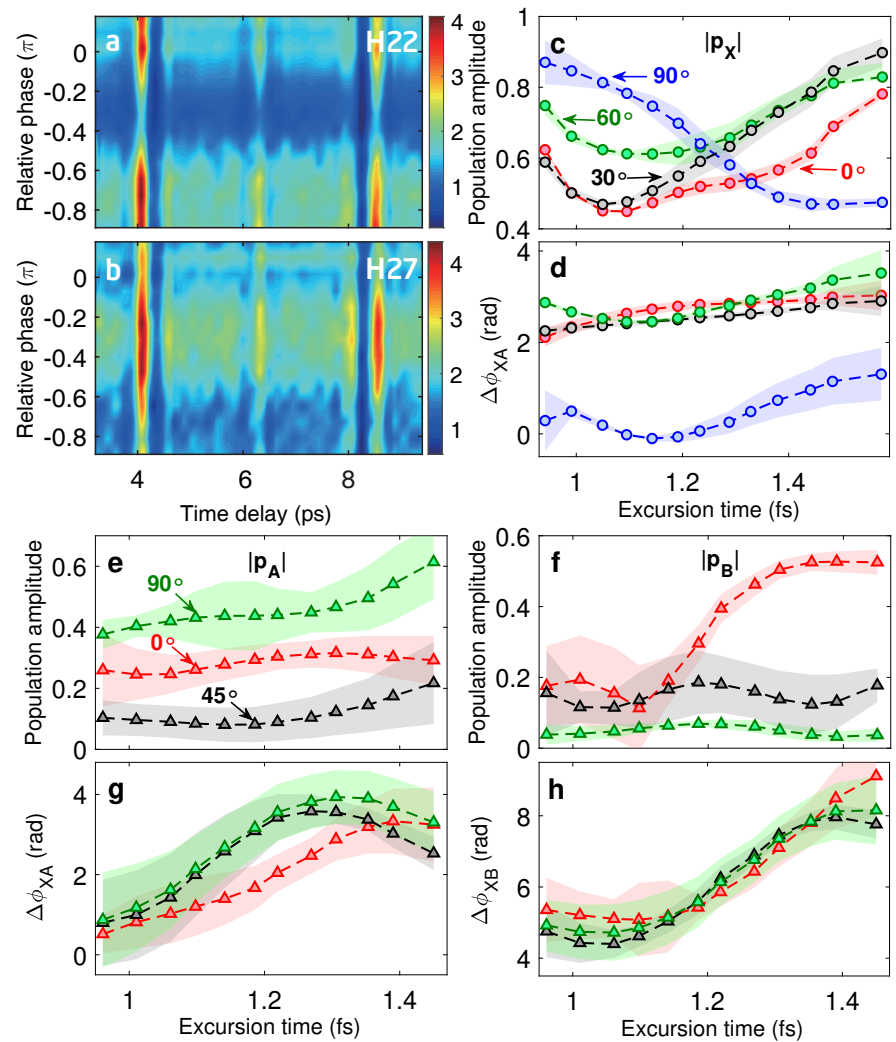

FIG. 2: Two-color HHS scheme for probing molecular charge migration in $\mathbf{N}_{2}$ and $\mathbf{C O}_{2}$. a-b, Measured harmonic signals of $\mathrm{H} 22$ and $\mathrm{H} 27$ in the two-color experiment of $\mathrm{N}_{2}$. Measurements at different relative phases of the two-color lasers provide sufficient data sets for resolving the multichannel electronic dynamics. c-d, Reconstructed population amplitude of the $\tilde{X}$ state (c) and the relative phase between the $\tilde{X}$ and $\tilde{A}$ states (d) in $\mathrm{N}_{2}$ for the alignment angles of $0^{\circ}$ (red circles), $30^{\circ}$ (black circles), $60^{\circ}$ (green circles), and $90^{\circ}$ (blue circles), respectively. Each circle represents the excursion time of a single harmonic order, as depicted in Fig. 1(b). e-f, Reconstructed population amplitudes of the $\tilde{A}$ state (e) and $\tilde{B}$ state (f) in $\mathrm{CO}_{2}$ for the alignment angles of $0^{\circ}$ (red triangles), $45^{\circ}$ (black triangles), and $90^{\circ}$ (green triangles). g-h, Reconstructed relative phases between the $\tilde{X}$ and $\tilde{A}(\mathbf{g})$ and $\tilde{X}$ and $\tilde{B}(\mathbf{h})$ states in $\mathrm{CO}_{2}$ for the above three angles. These amplitudes and phases retrieved for each harmonic order are used to construct the wave packet of the hole dynamics of the cations.

\section{Retrieval of single-molecule dipole}

For a parallel configuration of the pump and probe polarizations, the time-dependent harmonic signal is given by $[18,19]$

$$
S(\omega, \tau)=\left|\int_{0}^{\pi} D(\omega, \theta) \rho(\theta, \tau) \sin \theta d \theta\right|^{2},
$$



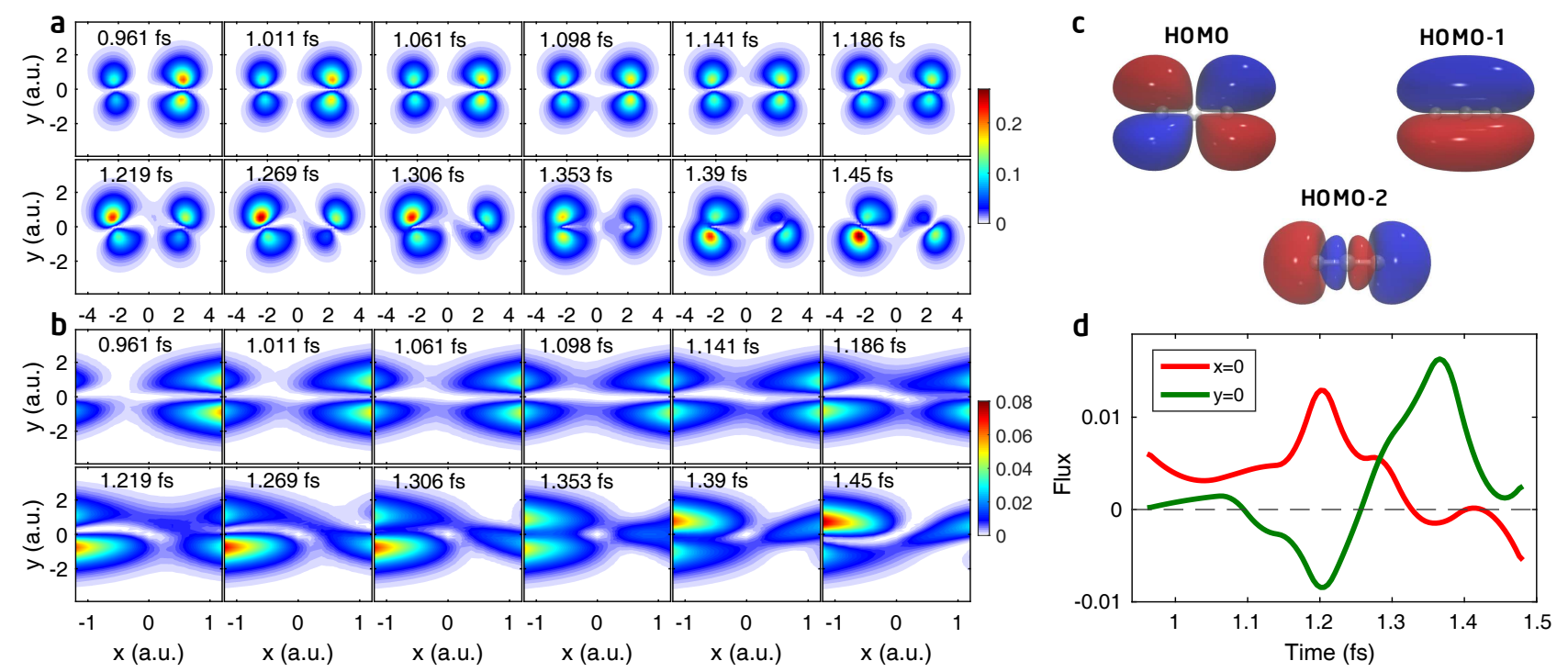

FIG. 3: Reconstruction of attoscoend charge migration in $\mathbf{C O}_{2}^{+}$. a, Snapshots of the reconstructed hole densities for the alignment angle of $0^{\circ}$. $\mathbf{b}$, Enlarged views of the results in $\mathbf{a}$ in the range from $\mathrm{x}=-1.2$ a.u. to $\mathrm{x}=1.2$ a.u.. c, HOMO, HOMO-1, and HOMO-2 orbitals of $\mathrm{CO}_{2}$ molecule. d, Flux of charge density crossing $\mathrm{x}=0$ a.u. (red) and $\mathrm{y}=0$ a.u. (green) planes. Positive value means charge migration from $+\mathrm{x}(+\mathrm{y})$ side to $-\mathrm{x}(-\mathrm{y})$ side.

where $\theta$ is the alignment angle of the molecule, $\tau$ is the pump-probe delay, $D(\omega, \theta)$ is the fixedangle-dependent total dipole moment from the single-molecule response, and $\rho(\theta, \tau)$ is the angular distribution of molecules at delay $\tau$. In our experiment, $\rho(\theta, \tau)$ is determined with method in [29]. Note that perfect molecular alignment can not be achieved in experiment. Our simulations show that single-molecule harmonic amplitudes and phases deviate substantially from the angle-averaged ones (see Supplementary Note 3 ).

To obtain molecular-frame electron dynamics, the first and foremost step is to decode angledependent single-molecule dipole moment $D(\omega, \theta)$ (including both amplitude and phase) from the measured harmonic signals. However, to solve this inverse problem directly is rather difficult since Eq. (1) is extremely nonlinear and is ill-posed. Here, we introduce ML to this problem. The modern ML algorithm has been demonstrated to have remarkable abilities in characterizing complex sets of data with a high degree of accuracy, and has been widely utilized in genetics [30], condensed-matter physics [31], and material science [32]. We show here that it can effectively deal with the complicated decoding in HHS (see Methods and Supplementary Note 4).

The second step of our reconstruction is to disentangle the multichannel contributions from the total single-molecule dipole moment $D(\omega, \theta)$ obtained in the first step. Driven by a one-color 
800-nm probe pulse, only one set of $D(\omega, \theta)$ can be obtained for each harmonic order which is insufficient for the reconstruction. To overcome this problem, we employed an additional parallel two-color laser field to generate harmonics. This two-color field consists of an intense 800-nm fundamental field and a weak second-harmonic $(\mathrm{SH})$ field. The $\mathrm{SH}$ field is weak $\left(\sim 2 \times 10^{-3}\right.$ of the fundamental field) such that it barely alters the electron dynamics of the molecular ion (see Supplementary Note 5). Measurement of harmonics at different relative phases between the fundamental and SH fields replenishes the additional data set needed to retrieve multichannel contributions in the second step. Figures 2(a,b) show the time-dependent signals of H22 and H27 from $\mathrm{N}_{2}$ measured as a function of the relative phase of the two-color laser fields. One can see that the modulation of HHG intensity depends sensitively on the relative phase, and different harmonic orders present different dependences. Applying the ML-based reconstruction procedure to each harmonic order, we can obtain the time-dependent complex mixing coefficients of the multiple orbitals of the molecular cation, for each fixed-in-space angle, at the excursion time when the recombination of that harmonic order occurs, as illustrated in Fig. 1(b).

Figure 2(c) shows the reconstructed population amplitudes of the ground $\tilde{X}$ state of the $\mathrm{N}_{2}^{+}$ion for four specific alignment angles versus the excursion time. The circles indicate the data extracted from the experiment for harmonics from H15 to H27 (including even and odd orders). Figure 2(d) shows the reconstructed relative phase between the $\tilde{X}$ and $\tilde{A}$ states. With these parameters, a complex-valued time-dependent wave packet from the two holes $\tilde{X}$ and $\tilde{A}$ can be constructed for $\mathrm{N}_{2}^{+}$.

We have also carried out the measurements for $\mathrm{CO}_{2}$ molecules. Harmonic spectra of $\mathrm{CO}_{2}$ involves three electronic states of the ion, the ground $\tilde{X}$ (HOMO), the first $\tilde{A}$ (HOMO-1) and second $\tilde{B}$ (HOMO-2) excited states [21, 22]. Using the same procedure as for $\mathrm{N}_{2}$, the reconstructed populations and relative phases of these three ion states are shown in Figs. 2(e,f) and Figs. 2(g,h), respectively. With these parameters, a complex-valued time-dependent wave packet from the three holes $\tilde{X}, \tilde{A}$ and $\tilde{B}$ can be constructed for $\mathrm{CO}_{2}^{+}$. To evaluate our reconstructions, we have carried out calculations based on the time-dependent density functional theory (TDDFT) to simulate the parameters we obtained. The theoretical calculations are in "reasonable" agreement with the reconstructions (see Supplementary Note 5).

\section{Filming attosecond charge migration}

To visualize the hole dynamics, we have calculated the modulus square of the wave packet versus time for $\mathrm{N}_{2}^{+}$and $\mathrm{CO}_{2}^{+}$with the data in Figs. 2(c-h). To quantify attosecond charge migration 

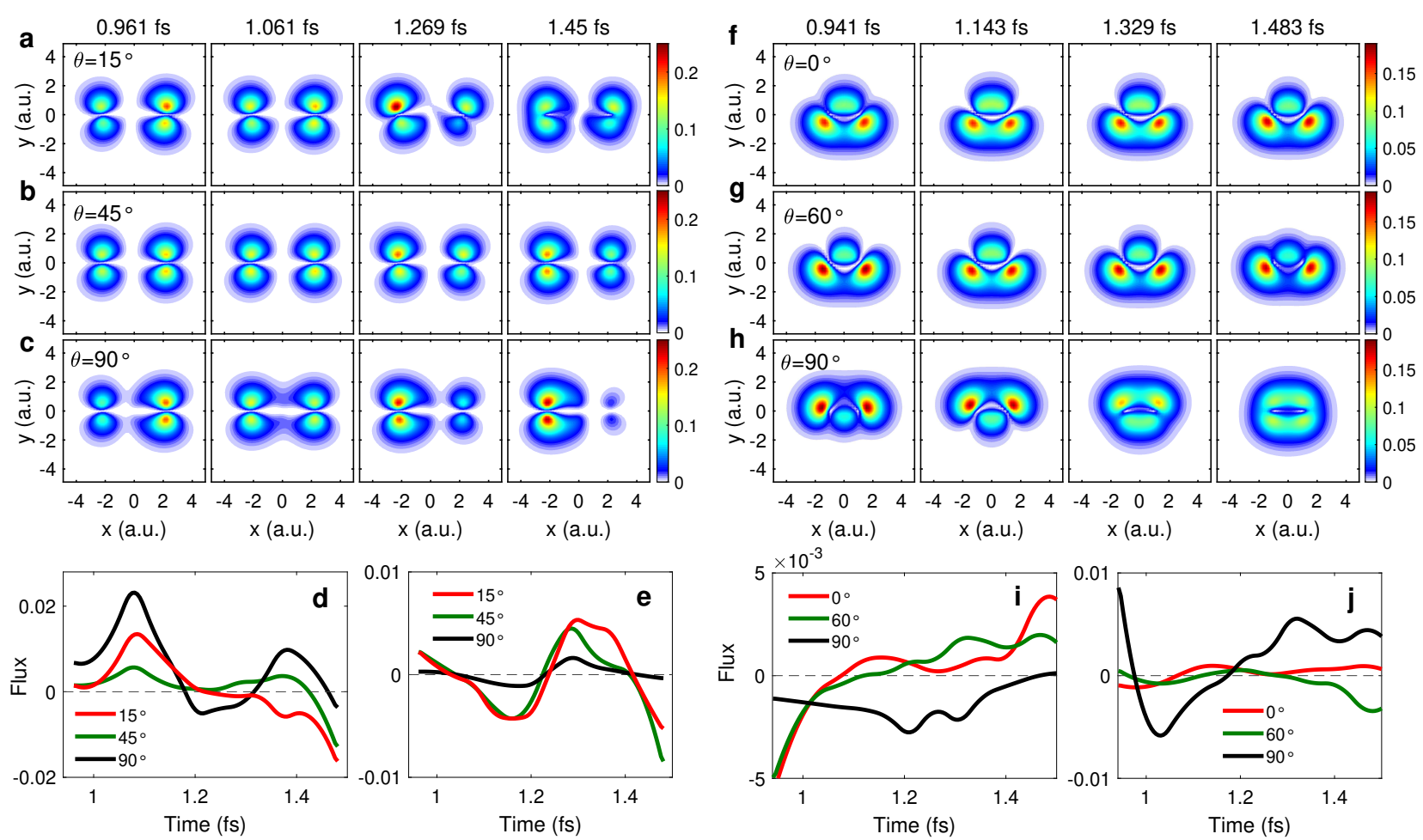

FIG. 4: Alignment-angle-dependent attoscoend charge migration in $\mathbf{N}_{2}^{+}$and $\mathbf{C O}_{2}^{+}$. a-c, Snapshots of the reconstructed hole densities in $\mathrm{CO}_{2}^{+}$for the alignment angles of $15^{\circ}, 45^{\circ}$ and $90^{\circ}$, respectively. d, Flux of charge density crossing the plane of $\mathrm{x}=0$ a.u. for the three angles in a-c. e, Same as $\mathbf{d}$, but for the $\mathrm{y}=0$ a.u. plane. Positive value means charge migration from $+\mathrm{x}(+\mathrm{y})$ side to $-\mathrm{x}(-\mathrm{y})$ side. $\mathbf{f}-\mathbf{h}$, Snapshots of the reconstructed hole densities in $\mathrm{N}_{2}^{+}$for the alignment angles of $0^{\circ}, 60^{\circ}$ and $90^{\circ}$, respectively. i, Flux of charge density crossing the plane of $\mathrm{x}=-1.5$ a.u. for the three angles in $\mathbf{f}-\mathbf{h}$. $\mathbf{j}$, Same as $\mathbf{i}$, but for the $\mathrm{y}=0$ a.u. plane.

processes, we first examine hole dynamics of $\mathrm{CO}_{2}^{+}$when the fixed-in-space molecule is aligned parallel to the laser polarization axis. In Fig. 3(c), we plot the relevant molecular orbitals to help understanding. Figure 3(a) presents hole densities extracted at the recombination times of harmonics $\mathrm{H} 15$ to $\mathrm{H} 26$. Here, $\mathrm{t}=0$ is when electron is born. In the figures, the frames are separated by steps of about 50 as. At a quick glance, we can see substantial hole migration from $+\mathrm{x}$ side to the -x side over the 0.5 fs duration. To take a closer look at how the hole migrates, in Fig. 3(b) we zoom in the region of $\mathrm{x}=[-1.2$ a.u., 1.2 a.u.]. In the first $0.25 \mathrm{fs}$, we can see that the hole density coalesces in the middle region along the $\mathrm{x}$-axis slowly from the $+\mathrm{x}$ side to the $-\mathrm{x}$ side, but in the second half, the pace turns faster where at later time we can see some density is moving back to the $+\mathrm{x}$ side. In addition, the migration seems to deplete density from the 4 th $\rightarrow 2$ nd $\rightarrow 3$ rd $\rightarrow 1$ st $\rightarrow 2$ nd quadrants. In fact, its time evolution is more like a counterclockwise swirling than a direct migration. To 
observe the continuous evolution of hole density, we have constructed movies at steps of 10 as by interpolating the mixing coefficients in the complex wave packets shown in Figs. 2(e-h) (see Supplementary movie 1).

Since quantum mechanics can predict single hole behavior only statistically, it is inappropriate to associate such "movement" with trajectory or speed. It is more proper to think such movement like that of a flock of migrating birds. Each bird has its own mind and there is nothing to govern the trajectory of each bird, yet the flock will move on. In the presence of an external disturbance, the movement of the flock will change. So is the migration of an electron or a hole in the presence of the laser field. To describe the "movement" of charge density of a quantum system, consider the equation of continuity in quantum mechanics, $\frac{\partial \rho_{e}}{\partial t}+\nabla \cdot \mathbf{J}=0$, where $\rho_{e}$ is the probability density and $\mathbf{J}$ is the probability current density. By calculating the latter quantum mechanically from the complex hole wave packet, we can then calculate the total flux crossing the $\mathrm{x}=0 \mathrm{a}$.u. plane at each time instant, as shown by the red line in Fig. 3(d). We have confirmed that the total flux change is equal to the rate of change of the total charge density. Likewise, the total flux crossing the $y=0$ a.u. plane can be calculated and shown as the green line. The total flux varies widely versus time, implying the statistical nature of the probability current density.

Our reconstruction method for each harmonic obtains the occupation amplitude and phase for all angles of each fixed-in-space molecules. In Figs. 4 (a-c), we show a sample of hole density plots for alignment angles of $15^{\circ}, 45^{\circ}$ and $90^{\circ}$, respectively. For the same 0.5 fs duration, we can clearly see that the hole density do undergo significant change, and at the same time, the hole density for different angles does show large difference. This is not surprising since for a fixed-in-space molecule, changing the direction of the laser polarization will drive the electrons in the molecule quite differently. These results reflect that one should not compare single-molecule harmonic phase and amplitude with those derived from the experiment directly without considering integration or average over the angular distribution of molecules. In Figs. 4(d,e) we also calculated the flux across the $\mathrm{x}=0$ a.u. and $\mathrm{y}=0$ a.u. planes, respectively, for the three angles. In the Supplementary movies $2,3,4$ we show the movies of hole migration for these three angles.

In Figs. 4(f-h), we show the reconstructed hole densities in $\mathrm{N}_{2}$ at several selected excursion times for the alignment angles of $0^{\circ}, 60^{\circ}$ and $90^{\circ}$, respectively. The hole densities are symmetric about $\mathrm{x}=0$ a.u. plane for all alignment angles because of the symmetry of the HOMO and HOMO-1 orbitals of $\mathrm{N}_{2}$. At $0^{\circ}$ and $60^{\circ}$, only small migration can be seen. This can be understood from Figs. 2(c,d), where the population amplitude does not change much versus time and the relative phase stays nearly close to $\pi$. On the other hand, at $90^{\circ}$, the population drops significantly within 
the 0.5 fs excursion time, thus hole density evolves more dramatically. These results are consistent with the time dependence of total flux across the $\mathrm{x}=-1.5$ a.u. and $\mathrm{y}=0$ a.u. planes, respectively, as shown in Figs. 4(i,j). Note that the hole density is symmetric about $\mathrm{x}=0$ a.u. plane, thus the flux crossing the $\mathrm{x}=0$ a.u. plane is very small. Hole migration for $\mathrm{N}_{2}$ has also been constructed (see Supplementary movies 5-8).

Comparing to results previously reported by Kraus et al. [17], we are able to make movies of charge migration at steps of 10 as, for molecules at all alignment angles. This is because we were able to retrieve single molecule complex dipole at each fixed-in-space angle and probe charge migration at recombination times for both even and odd harmonics at every 50 as, instead of 100 as if only odd harmonics are measured.

\section{DISCUSSION}

In summary, we have used a ML-based HHS method to construct movies of charge migration in a molecular ion at its natural timescale of 10 as to follow the change of hole density during the time between electron is removed till it has recombined with the ion to emit harmonics. We presented charge migration at the most fundamental level for each single fixed-in-space molecule at all alignment angles from the experimental HHG spectra. The method presented here is general and can be extended to other molecules. In contrast to the conventional belief that attosecond electron dynamics should be probed with attosecond-pump-attosecond-probe scheme, this work suggests that HHS is the simplest route to probing electron dynamics involving valence electrons of molecules. Using HHS, a unique one-to-one time-energy relation is established through constructive interference of harmonic emissions from a multi-cycle long driving laser. Both the technology and methods of analysis for studying attosecond charge migration are now available. Looking ahead, it is highly desirable to extend our method to more complex molecules, to explore how the charge migration depends on the structure of molecules.

\section{METHODS}

Experimental methods. Our experiment is carried out by using a commercial Ti:sapphire laser system (Legend Elite-Duo, Coherent, Inc.), which delivers 35-fs, 800-nm laser pulses at a repetition rate of $1 \mathrm{kHz}$. The output laser is split into a pump and a probe pulse. The pump pulse with 
moderate intensity is used to induce nonadiabatic alignment of molecules along its polarization. The intense probe pulse has been used either directly (one-color scheme) or remoulded to a parallel two-color laser field (see Supplementary Note 1) to interact with the aligned molecules to generate high-order harmonics. The pump and probe pulses are parallel in the polarization. A motorized delay line is installed in the arm of the pump pulse to adjust the pump-probe delay. These two pulses are focused to a supersonic gas jet by a spherical mirror $(\mathrm{f}=250 \mathrm{~mm})$. The gas jet is placed $2 \mathrm{~mm}$ after the laser focus to ensure good phase matching for short-trajectory harmonics, and the backing pressure is maintained at 0.8 bar. The generated high harmonics are detected by a homemade flat-field soft x-ray spectrometer. In the one-color experiment, the harmonic signals measured at different pump-probe delays [Fig. 1(d) and Supplementary Fig. 2(e)] are used to identify the multiple orbitals effect in HHG. In the two-color experiment, the time-dependent harmonic signals are measured at various relative phases of the two-color laser field, providing a two-dimensional data set for decomposing the multiple orbital contributions in HHG process.

Reconstruction methods. Our reconstruction procedure has two steps. The first step is to retrieve single-molecule dipole moment from the measured time-dependent HHG signals. HHG from aligned molecular ensemble is expressed as Eq. (1). For a given harmonic order, Eq. (1) can be expanded as $[33,34]$

$$
\begin{aligned}
S(\tau) & =\left[\int_{0}^{\pi} D(\theta) \rho(\theta, \tau) \sin \theta d \theta\right]^{*}\left[\int_{0}^{\pi} D(\theta) \rho(\theta, \tau) \sin \theta d \theta\right] \\
& =\int_{0}^{\pi} \int_{0}^{\pi} D^{*}\left(\theta_{1}\right) D\left(\theta_{2}\right) \rho\left(\theta_{1}, \tau\right) \rho\left(\theta_{2}, \tau\right) \sin \theta_{1} \sin \theta_{2} d \theta_{1} d \theta_{2} .
\end{aligned}
$$

Let

$$
\begin{gathered}
R\left(\theta_{1}, \theta_{2}\right)=\operatorname{Re}\left[D^{*}\left(\theta_{1}\right) D\left(\theta_{2}\right)\right], \\
\rho\left(\theta_{1}, \theta_{2}, \tau\right)=\rho\left(\theta_{1}, \tau\right) \rho\left(\theta_{2}, \tau\right) \sin \theta_{1} \sin \theta_{2},
\end{gathered}
$$

and discretize $\theta_{1}, \theta_{2}$ in the range of $[0, \pi]$ with a step of $0.01 \mathrm{rad}$, the $S(\tau)$ then can be rewritten as

$$
S(\tau)=\sum_{p} \sum_{q} R\left(\theta_{1}^{p}, \theta_{2}^{q}\right) \rho\left(\theta_{1}^{p}, \theta_{2}^{q}, \tau\right) d \theta_{1} d \theta_{2}
$$

Note that in Eq. (3), the imaginary part of $D^{*}\left(\theta_{1}\right) D\left(\theta_{2}\right)$ is omitted due to its asymmetry upon the exchange of $\theta_{1}$ and $\theta_{2}$, which will vanish after the convolution. In Eqs. (2) and (4), the 
molecular axis distribution $\rho(\theta, \tau)$ and therefore $\rho\left(\theta_{1}, \theta_{2}, \tau\right)$ can be determined from the one-color experiment with the method in [29]. To retrieve single-molecule dipole moment $D(\theta)$, we first solve the matrix $R\left(\theta_{1}, \theta_{2}\right)$ according to Eq. (5). Here, we utilize a widely-used ML algorithm, sparse representation [35], to do the retrieval. In the reconstruction, we first build a dictionary matrix for $S(\tau)$ by expanding the $R$ matrix with a series of two-dimensional Legendre polynomial basis functions. The expansion coefficients then can be solved with the LASSO regression [36] by minimizing the $l_{1}$-norm of the coefficient vector and also the difference between the reproductions and the measurements. With $R\left(\theta_{1}, \theta_{2}\right)$ retrieved, we can obtain the amplitude and phase of the dipole moment $D(\theta)$ in terms of the derivations of Eq. (3). The validity and robustness of this algorithm have been validated by simulation experiments (see Supplementary Note 4). Applying this procedure to measurements at different relative phases $\alpha$ of the two-color laser field, we can get a set of $D(\theta ; \alpha)$ for each harmonic order.

The second step of the reconstruction is to decompose the multichannel contributions from the total dipole moment $D(\theta ; \alpha)$ obtained in the first step. In molecular HHG, the total dipole moment $D(\omega, \theta)$ is a coherent superposition of the induced dipole moment of each emission channel, i.e.,

$$
D(\omega, \theta)=\sum_{i} D_{i}(\omega, \theta)
$$

where $D_{i}(\omega, \theta)$ is the induced dipole moment of each emission channel. The subscript $i$ denotes the involved molecular orbitals (molecular ion states) during the HHG process. Consider the initially populated molecular ion state $i, D_{i}(\omega, \theta)$ is given by

$$
D_{i}(\omega, \theta)=d_{i o n}^{i}(\omega, \theta) a_{a c c}(\omega) d_{r e c}^{i}(\omega, \theta)
$$

where $d_{i o n}^{i}(\omega, \theta)$ and $d_{r e c}^{i}(\omega, \theta)$ are the transition dipole moments related to the ionization and recombination steps, respectively. $a_{a c c}(\omega)$ denotes the propagation amplitude of the electron wave packet in the continuum. $d_{i o n}^{i}(\omega, \theta)$ can be expressed as

$$
\begin{aligned}
d_{i o n}^{i} & =\left\langle\Psi_{0}(N)|\tilde{D}| \Phi_{i}(N-1) \chi_{k}\right\rangle \\
& =\left\langle\psi_{i}|r| \chi_{k}\right\rangle
\end{aligned}
$$

Here $\Psi_{0}(N)$ is the ground state of the N-electron molecule, $\Phi_{i}(N-1)$ is the ground state or excited state of the (N-1)-electron molecular ion, where a molecular orbital $\psi_{i}$ has been removed to a continuum state $\chi_{k}$. Both many-electron wavefunctions $\Psi_{0}(N)$ and $\Phi_{i}(N-1) \chi_{k}$ are properly antisymmetrized and $\tilde{D}$ is the dipole operator from all the electrons. In obtaining Eq. 8(b), we 
assume that all the molecular orbitals are in the neutral and the ion does not change before and after ionization.

To calculate the recombination transition dipole, we take into account that the molecular ion has been modified by the laser field during the time interval between ionization and recombination to $\Phi_{i}^{\prime}(N-1)$, where

$$
\Phi_{i}^{\prime}(N-1)=\sum_{j} C_{i j}(\theta) \Phi_{j}(N-1) .
$$

Thus, the recombination dipole

$$
\begin{aligned}
d_{r e c}^{i} & =\left\langle\Phi_{i}^{\prime}(N-1) \chi_{k}|\tilde{D}| \Psi_{0}(N)\right\rangle \\
& =\sum_{j} C_{i j}(\theta)\left\langle\chi_{k}|r| \psi_{j}\right\rangle .
\end{aligned}
$$

Inserting Eq. (10) to Eq. (7), we obtain

$$
D_{i}(\omega, \theta)=\sum_{j} C_{i j}(\theta) d_{i o n}^{i}(\omega, \theta) a_{a c c}(\omega) d_{r e c}^{j}(\omega, \theta) .
$$

Eq. (11) implies that hole hopping occurs before recombination. The degree of hopping depends on laser parameter and the alignment angle $\theta$ of the molecule. With Eq. (11), the total dipole moment for HHG can be expressed as

$$
D(\omega, \theta)=\sum_{i j} C_{i j}(\theta) \bar{D}_{i j}(\omega, \theta)
$$

where

$$
\bar{D}_{i j}(\omega, \theta)=d_{i o n}^{i}(\omega, \theta) a_{a c c}(\omega) d_{r e c}^{j}(\omega, \theta)
$$

In our reconstruction, only the most relevant molecular orbitals $\psi_{j}$ are considered (that is, $\tilde{X}$ and $\tilde{A}$ states for $\mathrm{N}_{2}$, and $\tilde{X}, \tilde{A}$, and $\tilde{B}$ states for $\left.\mathrm{CO}_{2}\right)$. The dipole moment $\bar{D}_{i j}(\omega, \theta)$ is calculated under the experimental laser conditions. The complex-valued coefficients $C_{i j}(\theta)$ are directly associated to the electron dynamics in the molecular ion. To determine $C_{i j}(\theta)$, we have performed two-color experiment. Since the SH field in the two-color experiment is weak enough and hardly alters the laser-induced electron dynamics, the coefficients $C_{i j}(\theta)$ can be assumed to be independent of the relative phase $\alpha$. The coefficients $C_{i j}(\theta)$ are then retrieved from the dipole moment $D(\theta ; \alpha)$ obtained at different relative phases $\alpha$ by solving Eq. (12) with the genetic algorithm.

With the coefficients $C_{i j}(\theta)$ retrieved, we can further calculate the wave function of the molecular ion at the recombination instant as

$$
\psi_{\text {sum }}=\sum_{i} \gamma_{i}(\theta) \psi_{i}^{\prime}=\sum_{i} \sum_{j} \gamma_{i}(\theta) C_{i j}(\theta) \psi_{j}
$$


and the population coefficient of the molecular orbital $\psi_{j}$ as [17],

$$
p_{j}=\sum_{i} C_{i j}(\theta) \gamma_{i}(\theta),
$$

where $\gamma_{i}(\theta)$ is the initial population of the molecular ion state $i$, which is related to the alignmentangle-dependent ionization rate $\eta_{i}(\theta)$ by $\left|\gamma_{i}(\theta)\right|^{2}=\eta_{i}(\theta) / \Sigma_{i} \eta_{i}(\theta)$. In our work, the alignmentangle-dependent ionization rates of each molecular orbital are simulated by the MO-ADK theory $[37,38]$. Repeating the above procedure for different harmonic orders, we can then construct the hole dynamics in the ionized molecular ion according to time-frequency mapping underlying the HHG process.

\section{Acknowledgements}

We acknowledge fundings from National Key Research and Development Program of China (No. 2019YFA0308300, 2017YFE0116600), National Natural Science Foundation of China (No. 91950202, 12074136, 12021004, 11627809, 11934006). C.D.L. was supported by Chemical Sciences, Geosciences and Biosciences Division, Office of Basic Energy Sciences, Office of Science, U.S. Department of Energy under Grant No. DE-FG02-86ER13491.

Data availability. All the data that support the findings of this study are available from the corresponding author upon reasonable request.

Competing interests: The authors declare no competing interests.

\section{Author contributions}

P.F.L. and P.X.L. conceived this research. L.X.H., Y.Q.H., and P.W. performed the experiments. S.Q.S developed the reconstruction algorithm. L.X.H. and Y.Q.H. performed the simulations. C.D.L., L.X.H., and P.F.L. analyzed the data and wrote the manuscript. All the authors participated in the discussions.

[1] Cederbaum, L. S. \& Zobeley, J. Ultrafast charge migration by electron correlation. Chem. Phys. Lett. 307, 205-210 (1999).

[2] Calegari, F. et al. Charge migration induced by attosecond pulses in bio-relevant molecules. J. Phys. B: At. Mol. Opt. Phys. 49, 142001 (2016). 
[3] Kraus, P. M. \& Wörner H. J. Perspectives of attosecond spectroscopy for the understanding of fundamental electron correlations. Angew. Chemie Int. Ed. 57, 5228-5247 (2018).

[4] Calegari, F. et al. Ultrafast electron dynamics in phenylalanine initiated by attosecond pulses. Science 346, 336339 (2014).

[5] Remacle, F. \& Levine, R. D. An electronic time scale in chemistry. Proc. Natl. Acad. Sci. U.S.A. 103, 67936798 (2006).

[6] Kuleff, A. I., Lünnemann, S. \& Cederbaum, L. S. Electron-correlation-driven charge migration in oligopeptides. Chem. Phys. 414, 100105 (2013).

[7] Itatani, J., Levesque, J., Zeidler, D., Niikura, H., Pépin, H., Kieffer, J. C., Corkum, P. B. \& Villeneuve, D. M. Tomographic imaging of molecular orbitals. Nature 432, 867 (2004).

[8] Haessler, S. et al. Attosecond imaging of molecular electronic wavepackets. Nat. Phys. 6, 200 (2010).

[9] Li, W., Zhou, X., Lock, R., Patchkovskii, S., Stolow, A., Kapteyn, H. C. \& Murnan, M. M. Timeresolved dynamics in $\mathrm{N}_{2} \mathrm{O}_{4}$ probed using high harmonic generation. Science 322, 1207 (2008).

[10] Kraus, P. M., Rupenyan, A., \& Wörner, H. J. High-harmonic spectroscopy of oriented OCS molecules: emission of even and odd harmonics. Phys. Rev. Lett. 109, 233903 (2012).

[11] Corkum, P. B. Plasma perspective on strong field multiphoton ionization. Phys. Rev. Lett. 71, 1994 (1993).

[12] Lewenstein, M., Balcou, Ph., Ivanov, M., L'Huillier, A. \& Corkum, P. B. Theory of high-harmonic generation by low-frequency laser fields. Phys. Rev. A 49, 2117 (1994).

[13] Mairesse, Y. et al. Attosecond synchronization of high-harmonic soft x-rays. Science 302, 1540 (2003).

[14] Lein, M. Molecular imaging using recolliding electrons. J. Phys. B 40, R135R173 (2007).

[15] Baker, S. et al. Probing proton dynamics in molecules on an attosecond time scale. Science 312, 424 (2006).

[16] Lan, P. et al. Attosecond probing of nuclear dynamics with trajectory-resolved high-harmonic spectroscopy. Phys. Rev. Lett. 119, 033201 (2017).

[17] Kraus, P. M. et al. Measurement and laser control of attosecond charge migration in ionized iodoacetylene. Science 350, 790 (2015).

[18] He, L. et al. Real-Time Observation of Molecular Spinning with Angular High-Harmonic Spectroscopy. Phys. Rev. Lett. 121, 163201 (2018).

[19] He, Y. et al. Direct imaging of molecular rotation with high-order-harmonic generation. Phys. Rev. A 99, 053419 (2019).

[20] Yoshii, K., Miyaji, G. \& Miyazaki, K. Retrieving Angular Distributions of High-Order Harmonic Generation from a Single Molecule. Phys. Rev. Lett. 106, 013904 (2011).

[21] Smirnova, O., Mairesse, Y., Patchkovskii, S., Dudovich, N., Villeneuve, D., Corkum, P. \& Ivanov, M. Y. High harmonic interferometry of multi-electron dynamics in molecules. Nature 460, 972 (2009).

[22] Smirnova, O., Patchkovskii, S., Mairesse, Y., Dudovich, N. \& Ivanov, M. Y. Strong-field control and spectroscopy of attosecond electron-hole dynamics in molecules. Proc. Natl. Acad. Sci. U.S.A. 106, 
$16556(2009)$.

[23] Mairesse, Y., Higuet, J., Dudovich, N., Shafir, D., Fabre, B., Mével, E., Constant, E., Patchkovskii, S., Walters, Z., Ivanov, M. Y. \& Smirnova, O. High Harmonic Spectroscopy of Multichannel Dynamics in Strong-Field Ionization. Phys. Rev. Lett. 104, 213601 (2010).

[24] McFarland, B. K., Farrell, J. P., Bucksbaum, P. H. \& Gühr, M. High Harmonic Generation from Multiple Orbitals in $\mathrm{N}_{2}$. Science 322, 1232-1235 (2008).

[25] Le, A. T., Lucchese, R. R., Tonzani, S., Morishita, T. \& Lin, C. D. Quantitative rescattering theory for high-order harmonic generation from molecules. Phys. Rev. A 80, 013401 (2009).

[26] Lin, C. D., Le, A. T., Chen, Z., Morishita, T. \& Lucchese, R. Strong-field rescattering physics - selfimaging of a molecule by its own electrons. J. Phys. B: At. Mol. Opt. Phys. 43, 122001 (2010).

[27] Lin, C. D., Le, A. T., Jin, C. \& Wei, H. Attosecond and Strong-Field Physics: Principles and Applications. (Cambridge University Press, Cambridge, UK, 2018).

[28] Lin, C. D., Le, A. T., Jin, C. \& Wei, H. Elements of the quantitative rescattering Theory. J. Phys. B: At. Mol. Opt. Phys. 51, 104001 (2018).

[29] He, Y. et al. Measuring the rotational temperature and pump intensity in molecular alignment experiments via high harmonic generation. Opt. Express 28, 21182-21191 (2020).

[30] Libbrecht, M. W. \& Noble, W. S. Machine learning applications in genetics and genomics. Nat. Rev. Genet. 16, 321 (2015).

[31] Carrasquilla, J. \& Melko, R. G. Machine learning phases of matter. Nat. Phys. 13, 431 (2017).

[32] Raccuglia, P. et al. Machine-learning-assisted materials discovery using failed experiments. Nature 533, $73(2016)$

[33] Wang, X., Le, A., Zhou, Z., Wei, H. \& Lin, C. D. Theory of retrieving orientation-resolved molecular information using time-domain rotational coherence spectroscopy. Phys. Rev. A 96, 023424 (2017).

[34] Wang, B. et al. Retrieval of full angular- and energy-dependent complex transition dipoles in the molecular frame from laser-induced high-order harmonic signals with aligned molecules. Phys. Rev. A 101, 063417 (2020).

[35] Rubinstein, R., Bruckstein, A. M. \& Elad, M. Dictionaries for sparse representation modeling. Proceedings of the IEEE 98, 1045-1057 (2010).

[36] Tibshirani, R. Regression Shrinkage and Selection via the Lasso. Journal of the Royal Statistical Society. Series B. 58, 267-288 (1996).

[37] Tong, X. M., Zhao, Z. X. \& Lin, C. D. Theory of molecular tunneling ionization. Phys. Rev. A 66, $033402(2002)$.

[38] Tong, X. M. \& Lin, C. D. Empirical formula for static field ionization rates of atoms and molecules by lasers in the barrier-suppression regime. J. Phys. B: At. Mol. Opt. Phys. 38, 2593 (2005). 
Figures
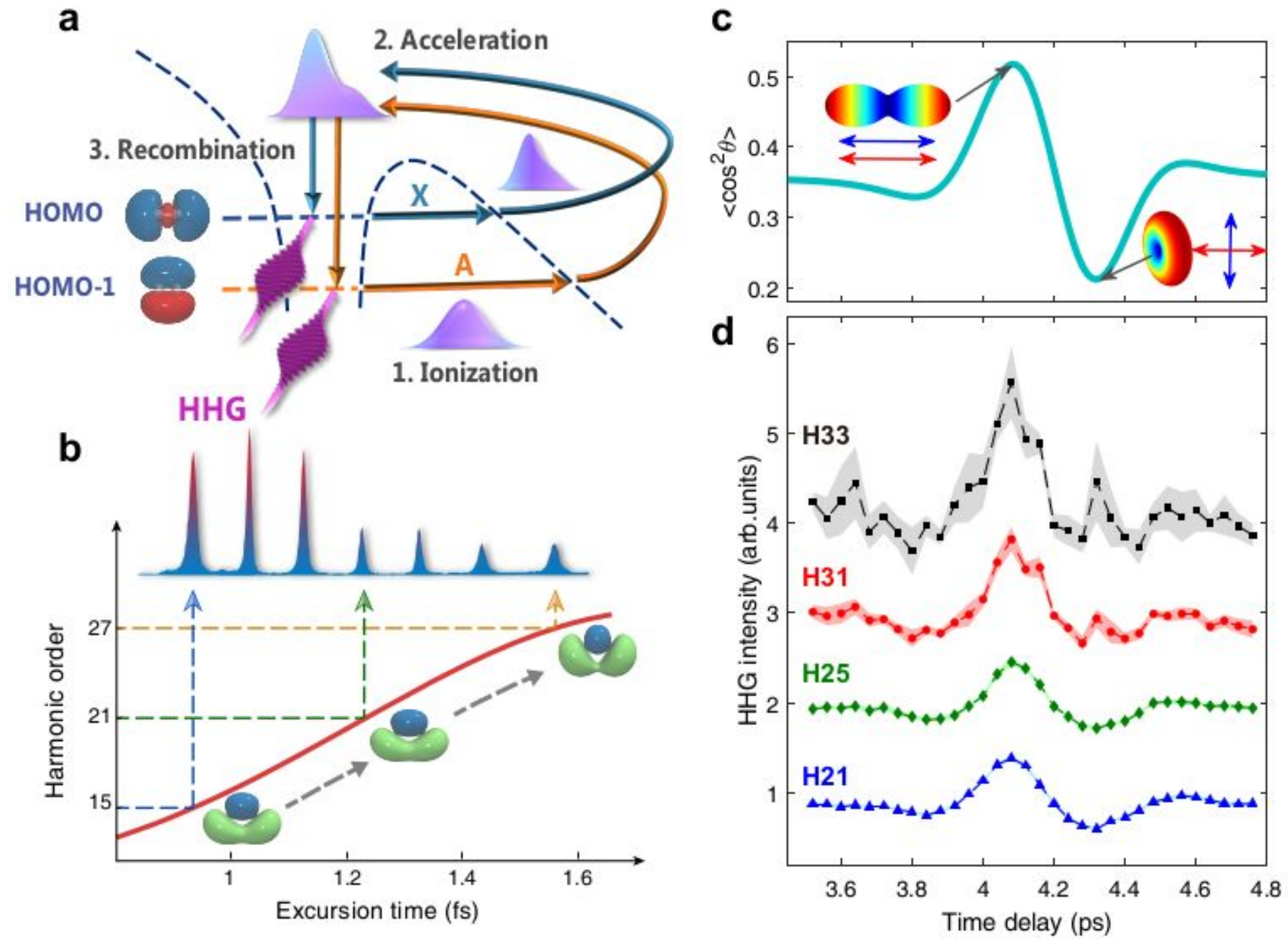

Figure 1

Due to technical limitations, figure captions can be found in the manuscript file. 

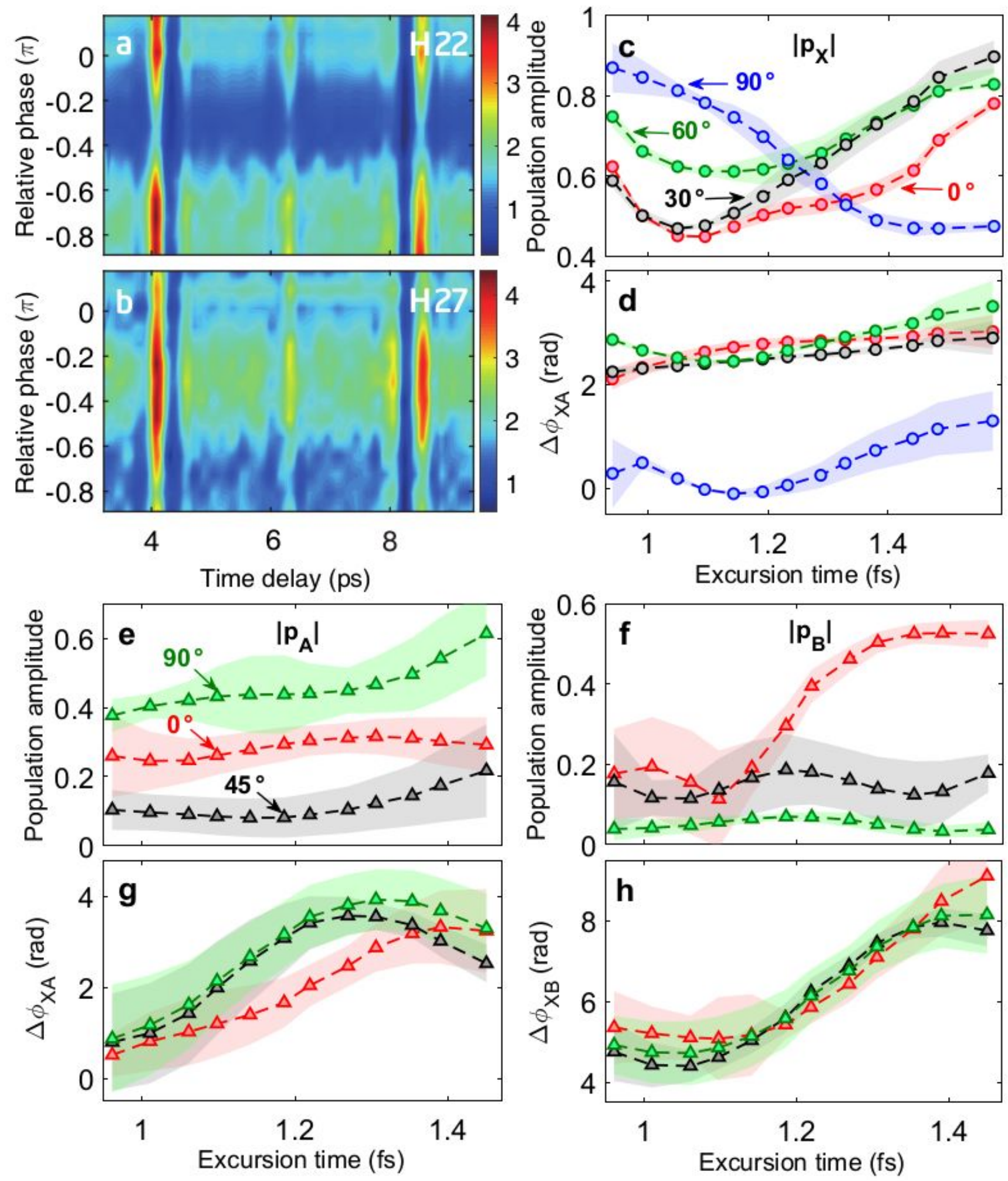

Figure 2

Due to technical limitations, figure captions can be found in the manuscript file. 

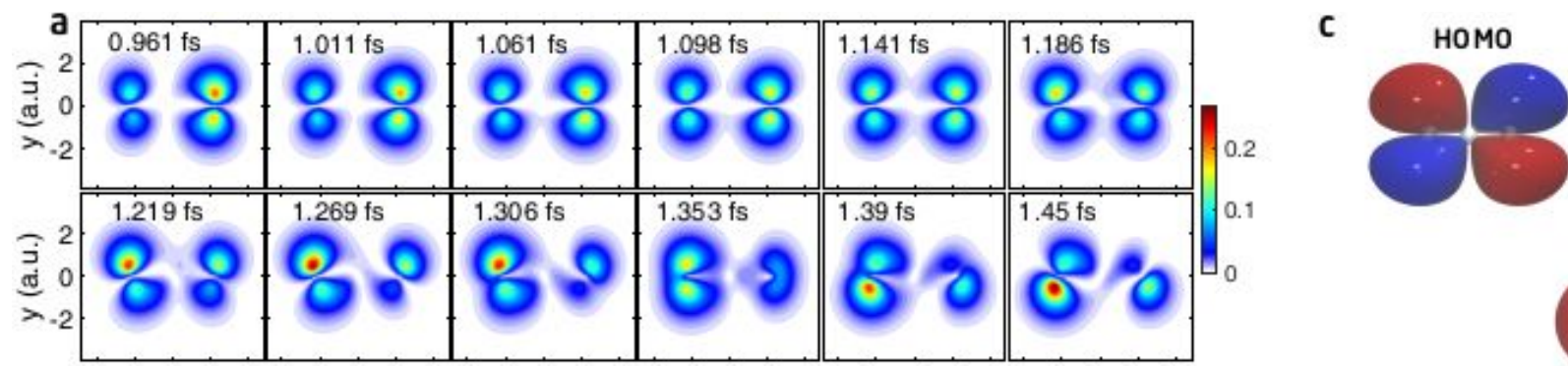

HOMO-1
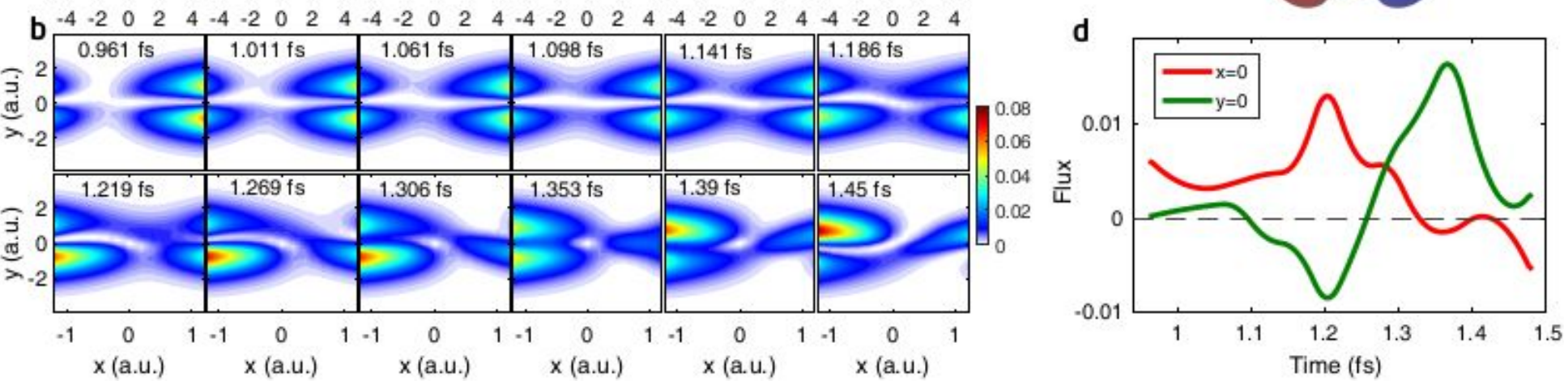

Figure 3

Due to technical limitations, figure captions can be found in the manuscript file.
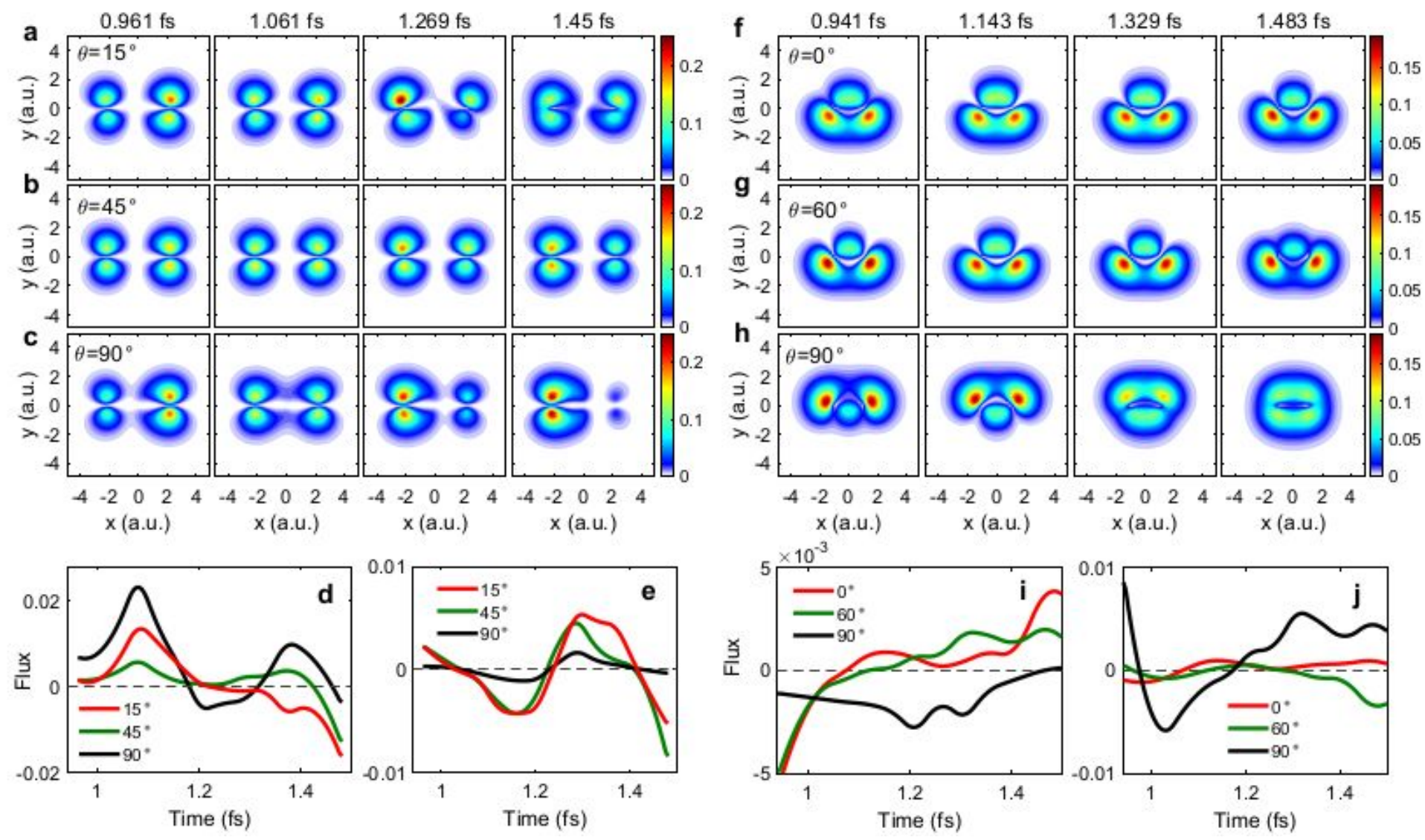

Figure 4

Due to technical limitations, figure captions can be found in the manuscript file. 


\section{Supplementary Files}

This is a list of supplementary files associated with this preprint. Click to download.

- CO290.mp4

- N290.mp4

- C0245.mp4

- C0215.mp4

- SupplementalMaterials.pdf

- N20.mp4

- N260.mp4

- CO20.mp4

- N230.mp4 